\title{
Editorial: Localization of Light in Randomly Disordered and Quasiperiodic Systems
}

\author{
Dan T. Nguyen ${ }^{1 *}$ and Axel Schülzgen ${ }^{2}$ \\ ${ }^{1}$ Corning Inc., Corning, NY, United States, ${ }^{2}$ CREOL, College of Optics and Photonics, University of Central Florida, Orlando, FL, \\ United States
}

Keywords: localization of light, randomly disordered, quasiperiodic systems, Anderson localization, aperiodic systems, principal modes, imaging fiber

Editorial on the Research Topic

Localization of Light in Randomly Disordered and Quasiperiodic Systems

Anderson localization, a very important phenomenon first discovered in solid state physics, has become the forefront of many research areas in physics. Photonic systems, randomly disordered or quasiperiodic in different dimensions, have emerged as a prominent platform for studying such localization phenomena. Recent discoveries in the field of localization of light are not only important for understanding the related physics but are also explored for a variety of applications including random lasing, perfect focusing, imaging, quantum communication, to name but a few. In the past decade, light localization phenomena have been theoretically and experimentally investigated in numerous systems, from arrays of randomly disordered waveguides to quasiperiodic photonics lattices and randomly disordered fibers using incoherent, coherent, or even quantum light sources.

Although Anderson localization in general, and localization of light in particular, have been at the forefront in different research areas in physics, little efforts have been made to attract readers who are not already familiar with the concept. Furthermore, several review papers focused on the fundamental aspects. It is our hope to provide a more balanced approach to the complex processes like localization of light including Anderson localization, making this Research Topic accessible for readers beyond the expert level and interesting for more application-oriented scientists. Several works on the light localization and its consequences in different photonic systems are included to provide a comprehensive picture of recent progress and present a better balance between basics research and application aspects, which has been missing so far.

Although localization of light in randomly disordered photonic systems can be well understood by Anderson's localization theory, similar localization effect have been increasingly realized in many quasiperiodic photonic systems which can be manipulated conveniently.

The Research Topic is divided in three themes.

Received: 19 January 2022 Accepted: 21 January 2022

Published: 18 February 2022

Citation:

- Anderson localization of light: This theme may include a tutorial review and new progress using randomly disordered photonics platforms.

- Localization of light in quasiperiodic photonic systems: A very recent area of research in which localization of light has been investigated in quasiperiodic photonic systems.

- Potential applications based on light localization: The aim is to present current studies on potential applications of light localization effects.

This Research Topic on the localization of light includes several review papers. The first one is a review of a decade of research on disordered Anderson localizing in optical fibers. The authors Mafi 
and Ballato, together with colleagues, had observed for the first time the transverse Anderson localization in an optical fiber with a random transverse refractive index profile. This started the development of a whole new class of optical fibers that guide light, not in a conventional core-cladding setting based on total internal reflection, but utilizing Anderson localization, where light can be guided at any location across the transverse profile of the fiber. These fibers have since been used successfully in high-quality endoscopic image transport. They also show interesting nonlinear and active (lasing) properties with promising applications. This review includes a brief history of these fibers with personal accounts of the events that led to their practical implementation. This review is followed by accounts of recent progress and future perspectives on science and applications of these fibers.

Another review paper on the field of transverse and quantum localization of light by leading experts Giordani et al.. This paper presents a comprehensive review of both theoretical and experimental works in this field. First, the authors present a general theory of Anderson Localization as an interference effect yielding a drastic reduction in diffusion-including complete hindrance-of wave packets such as sound, electromagnetic waves, and particle wave functions in the presence of strong disorder. In optics, this effect has been observed and demonstrated unquestionably only in dimensionally reduced systems. In particular, transverse localization (TL) occurs in optical fibers, which are disordered orthogonal to, and translationally invariant along, the propagation direction. The resonant and tube-shaped localized states act as micro-fiber-like single-mode transmission channels. Since the proposal of the first TL models in the early eighties, the fabrication technology and experimental probing techniques took giant steps forwards: TL has been observed in photo-refractive crystals, in plastic optical fibers, and also in glassy platforms, while employing direct laser writing it is now possible to tailor and "design" disorder. This review covers all these aspects that bring TL closer to applications such as quantum communication or image transport. Nonlinear optical phenomena in the TL regime, enabling steering of optical communication channels is also discussed. The authors further report on an experiment testing the traditional, approximate, way of introducing disorder into Maxwell's equations for the description of TL. The authors present a new theory, which does not involve an approximation and agrees with their experimental findings. Finally, they report on some quantum aspects, showing how a single-photon state can be localized in some of its inner degrees of freedom and how quantum phenomena can be utilized to secure a quantum communication channel.

The second theme is localization of light in quasiperiodic photonic systems. This very recent area of research is represented by "Wave Transport and Localization in Prime Number Landscapes" by Dal Negro et al.. The authors study the wave transport and localization properties in novel aperiodic structures that manifest the intrinsic complexity of prime number distributions in imaginary quadratic fields. In particular, the authors address structure-property relationships and wave scattering through the prime elements of imaginary quadratic fields (i.e., of their associated rings of integers) with class number one, which are unique factorization domains (UFDs). Their theoretical analysis combines the rigorous Green's matrix solution of the multiple scattering problem with the interdisciplinary methods of spatial statistics and graph theory analysis of point patterns to unveil the relevant structural properties that produce wave localization effects. The onset of a Delocalization Localization Transition (DLT) is demonstrated by a comprehensive study of the spectral properties of the Green's matrix and the Thouless number as a function of their optical density. Furthermore, they employ Multifractal Detrended Fluctuation Analysis (MDFA) to establish the multifractal scaling of the local density of states in these complex structures and discover a direct connection between localization, multifractality, and graph connectivity properties. Finally, the authors use a semi-classical approach to demonstrate and characterize the strong coupling regime of quantum emitters embedded in these novel aperiodic environments. Their study provides access to engineering design rules for the fabrication of novel and more efficient classical and quantum sources as well as photonic devices with enhanced light-matter interaction based on the intrinsic structural complexity of prime numbers in algebraic fields.

Finally, the theme of potential applications based on light localization is represented by several current research studies. In Nolan and Nguyen review the field of light localization and principal mode propagation in optical fibers. The capacity of optical fiber communications has grown exponentially since its implementation decades ago. Optical fiber amplifiers, wavelength division multiplexing, and coherent communications have all enabled discontinuous growth. Space division multiplexing has been investigated as the next discontinuity. Here tens of modes rather than a single mode are utilized in the transmission. In this study, random scattering due to index fluctuations within the optical fiber cause coupling among the modal channels thereby degrading signal transmission. However, it is demonstrated that principal mode transmission can overcome this limitation. Here a set of modes arrive localized at the fiber output unscattered. The authors review this methodology as it relates both optical communication capacity and the phenomenon of light localization. A theoretical and experimental characterization of these principal modes is presented.

In "Learning-Based Image Transport Through Disordered Optical Fibers With Transverse Anderson Localization", Zhao et al. review recent developments of Anderson localizing optical fibers and focus on the latest progress in deep-learning-based imaging applications using these fibers. Fiber-optic imaging systems play a unique role in biomedical imaging and clinical practice due to their flexibilities of performing imaging deep into tissues and organs with minimized penetration damage. Their imaging performance is often limited by the waveguide mode properties of conventional optical fibers and the image reconstruction method, which restrains the enhancement of image quality, transport robustness, system size, and illumination compatibility. The emerging disordered Anderson localizing optical fibers circumvent these difficulties by their intriguing properties of transverse Anderson localization of 
light, such as single-mode-like behavior, wavelength independence, and high mode density. To go beyond the performance limit of conventional system, there is a growing interest in integrating the disordered Anderson localizing optical fiber with deep learning algorithms.

Another research paper "Localization of Light in Photonics Lattices for All-Optical Representation of Binaries" by authors Nguyen et al. present a novel conceptual method for all optical representation of binary numbers that could be used for all-optical binary logic components in optical digital computing, as well as for other applications. The new concept is based on the effect of localization of light in specially designed binary photonics lattices whose central parts resemble the represented binaries, and localization occurs when periodicities of the lattices is broken. The proposed structures can be made with integrated photonics in on-chip devices that are highly programmable and controllable. Most significantly, the working principle of the novel method doesn't require nonlinear interaction between light and material, which is the most serious obstacle in the conventional method that uses optical transistors whose mechanism relies mainly on optical nonlinearity. The authors also discuss some technical challenges in developing the components.

We hope that the readers will enjoy the presentation of a plurality of different photonic platforms for generating novel light localization effects and will find interesting new ideas to utilize light localization for a variety of applications areas.

\section{AUTHOR CONTRIBUTIONS}

All authors listed have made a substantial, direct, and intellectual contribution to the work and approved it for publication.

Conflict of Interest: DTN was employed by Corning Inc.

The remaining author declares that the research was conducted in the absence of any commercial or financial relationships that could be construed as a potential conflict of interest.

Publisher's Note: All claims expressed in this article are solely those of the authors and do not necessarily represent those of their affiliated organizations, or those of the publisher, the editors and the reviewers. Any product that may be evaluated in this article, or claim that may be made by its manufacturer, is not guaranteed or endorsed by the publisher.

Copyright (c) 2022 Nguyen and Schülzgen. This is an open-access article distributed under the terms of the Creative Commons Attribution License (CC BY). The use, distribution or reproduction in other forums is permitted, provided the original author(s) and the copyright owner(s) are credited and that the original publication in this journal is cited, in accordance with accepted academic practice. No use, distribution or reproduction is permitted which does not comply with these terms. 